\title{
VIBRATIONS AND SOME ELECTRONIC EXCITATIONS OF ADSORBED ALKALI METAL MONOLAYERS
}

\author{
A. Hamawi, S.-A. Lindgren, C. Svensson and L. Walldén \\ Physics Department, Chalmers University of Technology \\ 41296 Göteborg, Sweden \\ (Received July 16, 1991; in revised form September. 3, 1991)
}

\begin{abstract}
We discuss briefly data obtained for adsorbed alkali metal monolayers with three different techniques (HREELS, UPS and SHG). The vibrational spectra provide support for the traditional picture of a fractionally charged adsorbate at low coverage and a neutral one at high coverage. We ascribe a vibrational overtone reported in previous work to contamination due to residual water vapour. UPS, uncharacteristically, gives little information of interest for low coverages except for substrates with a band gap and with a surface state in the gap. For $\mathrm{Na}$ on $\mathrm{Si}(100)$ we monitor the metallization of the surface region in the monolayer coverage range and observe metal induced gap states at higher coverages. For $\mathrm{Cs}$ on $\mathrm{Cu}(111)$ the optical second harmonic generation (SHG) shows a strong monolayer coverage dependence which we ascribe to optical transitions between surface bands and to surface barrier photoabsorption indicating the potential of SHG as a probe of the details of the surface electronic structure.
\end{abstract}

PACS numbers: 68.35. Ja, 73.20. Dx, 79.60. Gs, 78.65. Ez, 42.65. Ky

\section{Introduction}

We have used high resolution electron energy loss spectroscopy (IIREELS), optical second harmonic generation (SHG) and ultraviolet photoemission spectroscopy (UPS) to study adsorbed alkali metal monolayers. With HREELS the information looked for is the coverage dependence of the vibration frequency and of the loss intensity. If the vibrations are dipole excited the loss intensity gives the effective dynamic charge of the dipole. According to the traditional picture due to Gurney [1] of alkali metal adsorption one may expect the alkali adsorbate to vibrate like a fractionally charged particle and this is also the expectation based on the more recent calculations by Lang and Williams [2] and by Holmström [3]. At high coverage, by contrast, the adsorbate is expected to be a nearly neutral slab of metal. This is indicated for example by a strong adsorbate induced change 
of intensity at the Fermi edge of photoemission spectra [4] at high monolayer coverages. From the traditional picture one therefore expects that the adsorbate vibrations are easily excited at low coverage by the field produced by the incident electrons but not as easily at high coverage due to the screening of the field by the electron gas in the overlayer. As shown below the data provide no surprise in this respect. Less expected is that the frequency remains nearly constant independent of coverage in the coverage range where the vibrational loss can be observed in the present HREELS study. The desorption energy varies substantially with coverage $[5,6]$ and one might expect the vibration frequency to reflect this variation. Also unexpected is the observation by Astaldi et al. [7] of a strong overtone for both $\mathrm{Na}$ and $\mathrm{K}$ adsorbed on different $\mathrm{Cu}$ surfaces.

With UPS no information is obtained about the valence electron structure of the alkali metal adsorption systems at low coverage unless the substrate has a band gap with surface states. In that case it is found both for $\mathrm{Cu}(111)$ [8] and for $\mathrm{Be}(0001)$ [9] that adsorbed alkali metal atoms produce energy shifts of the substrate surface states. At high monolayer coverage one observes for metal substrates also a change of intensity at the Fermi edge and, if the substrate has a band gap, the emission of electrons out of overlayer states which are formed by valence electrons which are reflected back and forth between the vacuum barrier and the overlayer-substrate interface $[9,10]$. Below we will discuss our recent observations of such states for $\mathrm{Na}$ on $\mathrm{Si}(100)$. Since the overlayer is metallic at the coverages for which the overlayer states are observed the states are examples of metal induced gap states (MIGS).

Optical second harmonic generation (SHG) is less well established as a surface and adsorbate probe [11]. In the present work we show that for $\mathrm{Cu}(111) / \mathrm{Cs}$ the SH intensity shows a strong variation when the coverage is changed. We find that the structure observed when the SH intensity is drawn against the coverage can be explained in terms of transitions between electronic surface bands indicating the potential of SHG as a probe of surface electronic structure.

\section{Results and discussion}

\subsection{UPS}

\subsubsection{Observation of alkali valence resonances}

Photoelectron spectroscopy in general provides information about the valence electronic structure of chemisorption systems. It is interesting to note therefore that the technique has failed to provide any useful data at low monolayer coverage for alkali metal atoms adsorbed on metals except for metals with a band gap and with a surface state in the gap. From electronic structure calculations for alkali metal atom adsorption on the surface of a nearly free electron like metal substrate one would expect to record photoelectron energy spectra which reflect a partially filled, resonance broadened alkali valence electron state [2]. No such emission has been observed as far as we know. Recently a final state peak observed for $\mathrm{K}$ on $\mathrm{Ag}(111)$ was assigned to the empty part of the alkali valence resonance [12] but this structure has been observed previously and has been ascribed to the bulk 
electronic structure of the $\mathrm{Ag}(111)$ substrate [13]. It is so far only with Penning ionization spectroscopy that an emission peak ascribed to the valence electron resonance has been observed [14]. The interpretation of such spectra is however not straightforward. We have suggested as an alternative interpretation that the enhancement at the Fermi edge of the Penning ionization spectra may be produced by the work function change induced by the alkali metal adsorption [15].

\subsubsection{Metal substrates with band gap}

The interesting observations made so far by UPS at low alkali coverage is for metal substrates with a band gap and with a surface state in the band gap, namely for $\mathrm{Be}(0001)$ and $\mathrm{Cu}(111)$ substrates $[8,9]$. If the substrate has a band gap the resonance picture obviously does not apply since within the gap there are no states to resonate with. For both $\mathrm{Be}(0001)$ and $\mathrm{Cu}(111)$ the energy of a surface band shifts in a gradual manner as increasing numbers of alkali metal atoms are adsorbed on the surface. In both cases the shift is such that more electrons are accomodated by the surface band. The second important change of the surface state is that the tails of the state extend further into vacuum for the adsorbate covered surface. This is due to the reduced work function of the alkali covered surface. The surface band is thus modified by the adsorption such that it takes part in the charge transfer (by accomodating more electrons) and in the screening of the adsorbate (by shifting charge nearer to the adsorbate).

\subsubsection{Overlayer states and $k$-resolved photoemission}

Another interesting observation for substrates with a band gap is that there may appear discrete electron states due to the fact that the overlayer region acts as a quantum well. On one side there is a regular barrier, namely the surface potential barrier, while on the other side the electrons are totally reflected due to the substrate band gap. Such states have been observed via tunnelling $I-V$ characteristics for metal films thicker than around $100 \AA$ deposited on the insulating surface layer of an anodized Al substrate [16] and recently also by photoemission for much thinner films deposited on semiconductors [17] and metals [10, 18]. With photoemission the change of the electronic structure can be monitored in a layer by atomic layer fashion. Here one thus has the possibility of characterizing samples for studies of the thickness dependence layer by atomic layer of various other physical properties [19]. Of particular interest is also that the volume dispersion can now be measured with a potentially great accuracy by photoemission [20]. Earlier photoemission has been a $k_{\|}$-resolved spectroscopy. For thin films with overlayer states the perpendicular component of the wave vector can be obtained by repeated use of the phase condition satisfied by the overlayer states $\Phi_{B}+\Phi_{C}+2 k d=2 \pi m$, $m=0,1,2 \ldots \Phi_{B}$ and $\Phi_{C}$ are the phase changes at the vacuum barrier and the overlayer-substrate interface respectively and $d$ is the thickness of the overlayer. In the present we will limit the discussion to some recent results for $\mathrm{Na}$ overlayers on $\mathrm{Si}(100)$ obtained at low photon energies. 


\subsection{4. $\mathrm{Na}$ on $\mathrm{Si}(100)$}

When $\mathrm{Na}$ is deposited onto $\mathrm{Si}(100)$ and the sample is kept at low temperature, which means in our case that the sample is cooled by either liquid nitrogen or helium, the work function shows approximately the same coverage dependence as is typically observed upon alkali metal adsorption on noble and transition metal substrates with an accentuated minimum in the monolayer coverage range (Fig. 1). For a sample kept at RT there is only a very shallow minimum. Both these types

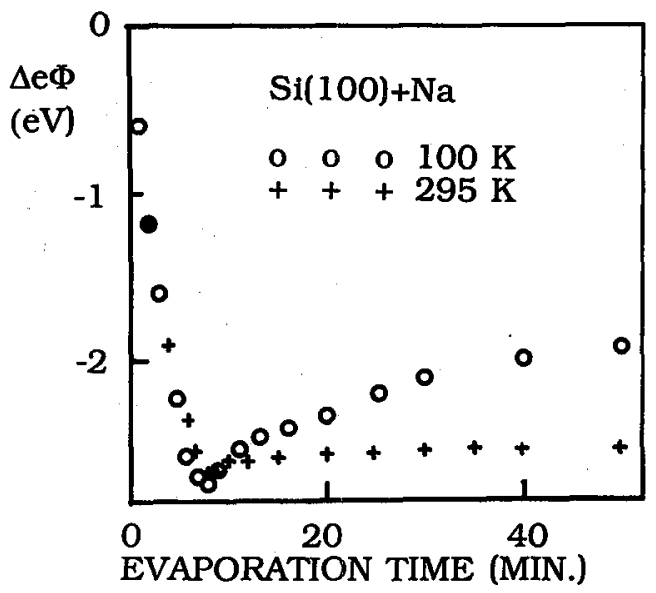

Fig. 1. Work function change versus $\mathrm{Na}$ deposition time for $\mathrm{Na}$ on $\mathrm{Si}(100)$ at $\mathrm{RT}$ and when the sample holder is cooled with $\mathbf{L N}_{2}$.

of coverages dependencies for $e \Phi$ have been observed previously for alkali metals adsorbed on $\mathrm{Si}(100)$ but it has not been observed that the sample temperature can make a difference for the work function variation [21-25]. When the Na evaporation is continued beyond the work function minimum there is a rapid increase of the intensity at the Fermi edge of photoemission spectra recorded at low photon energies (Fig. 2). This onset of emission intensity occurs at the same coverage at RT and at low temperature. The Fermi edge emission shows that the overlayer has a metallic character for coverages higher than the coverage at the work function minimum. The appearance of appreciable intensity at the Fermi edge is similar to that found for metal substrates $[4,8,26]$. The metallization of the $\mathrm{Si}(100)$ surface upon alkali adsorption has been studied also by other techniques $[27,28]$. It is only when the sample is kept at low temperature that emission due to overlayer states are observed. We ascribe this to a more homogeneous thickness for the films deposited at low temperature. At the evaporation rate chosen in the present series of measurements the work function minimum was reached after around min of evaporation time. An overlayer state is first observed after around 25 min deposition time (Fig. 3). As the evaporation is continued the energy of the peak changes in the manner expected for quantum well states for a thin $\mathrm{Na}$ film. When the peak first appears it is located at around $0.75 \mathrm{eV}$ below the Fermi edge. Since the sample 


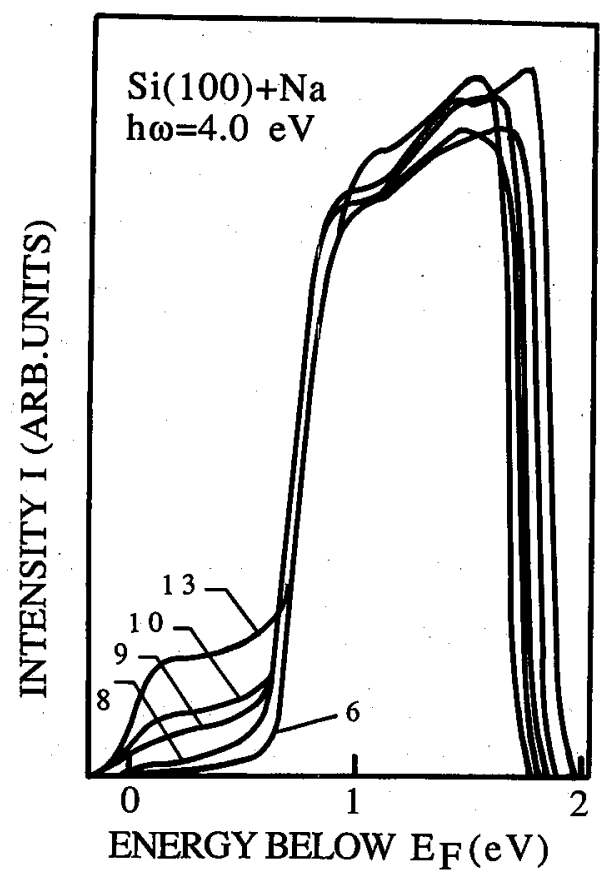

Fig. 2. Photoelectron energy spectra in the normal direction recorded at $4.0 \mathrm{eV}$ photon energy showing the onset of emission at the Fermi edge when $\mathrm{Na}$ deposition on $\mathrm{Si}(100)$ is continued beyond the work function minimum.

is $n$-type $(2 \Omega \mathrm{cm})$ it means that at this coverage the peak is close to the lower edge of the band gap. When the coverage is increased the state shifts into the gap. The state can then be characterized as a metal induced gap state (MIGS).

Clearly the peak due to the overlayer state emission resides on top of a background present already at lower coverages than necessary to produce the overlayer state. One possible explanation of this is that it is only patchwise that the overlayer thickness is homogeneous. Another possibility is that the overlayer states are formed in a layer which resides on top of a more ill defined intermediate metallic layer.

There is some uncertainty regarding the absolute $\mathrm{Na}$ coverages. We have earlier suggested that the one full monolayer is obtained after around $25 \mathrm{~min}$ evaporation. This may be wrong. At least it is in conflict with another of our recent observations, namely of a peak in the coverage dependence of the emission intensity at the Fermi edge after around $13 \mathrm{~min}$ evaporation time if the sample is cooled by liquid He. A similar peak, due to surface barrier emission, was observed for $\mathrm{Na}$ on $\mathrm{Cu}(100)$ at around full monolayer coverage [26]. While there is thus some uncertainty regarding the absolute coverage values the main result is thus that one may observe metal induced gap states for $\mathrm{Na}$ on $\mathrm{Si}(100)$. Such states 


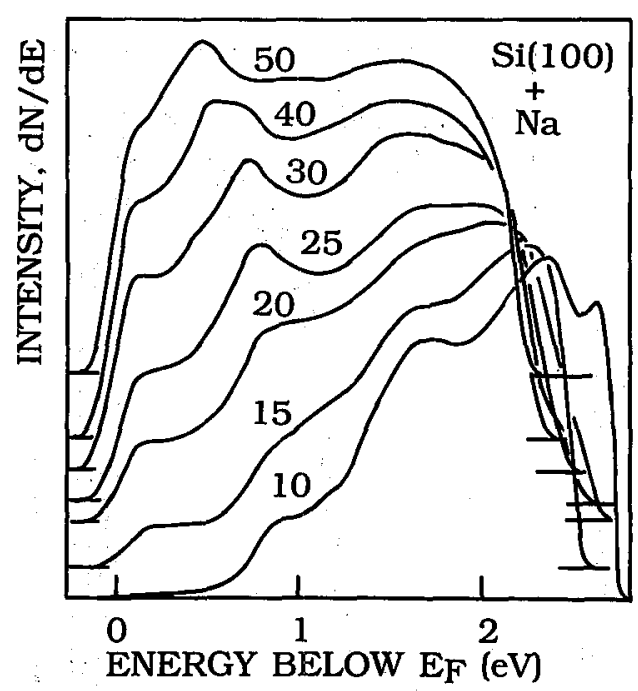

Fig. 3. Photoelectron energy spectra recorded at $4.9 \mathrm{eV}$ photon energy in the normal direction from $\mathrm{Si}(100)$ covered by different amounts of $\mathrm{Na}$ indicated by the evaporation times (in minutes) given in the diagram.

play a role in the theory of metal-semiconductor contacts but there have been few observations.

\subsection{HREELS for $\mathrm{Na}$ and $\mathrm{K}$ adsorbed on $\mathrm{Cu}(111)$}

Data from measurements on alkali adsorbates with high resolution electron energy loss spectroscopy (HREELS) at low incident energies (around $5 \mathrm{eV}$ ) have recently been briefly presented by Astaldi et al. [7] and by us [29]. The results agree to a large extent but differ on one interesting point. The results agree that the loss intensity is strong enough to be easily detected only for coverages below approximately half of a full monolayer. Our data show a maximum intensity at around $25 \%$ of full monolayer coverage. The loss intensity at low coverage corresponds to a dynamic dipole charge of around $0.4 e$ for $\mathrm{Na}$ and $0.5 e$ for $\mathrm{K}$. This is close to the value calculated by Lang and Williams from the slope $\mathrm{d} \mu / \mathrm{d} z$ of the adsorbate induced dipole moment with respect to the distance $z$ of the adsorbate from the surface of the metal which by them is represented by an $r_{s}=2$ jellium [2]. The loss intensity thus shows the coverage dependence expected from the model of a partly ionic adsorbate at low coverage and a neutral one with metal character at high monolayer coverage.

The vibration frequency remains nearly independent of coverage in the coverage range of our observations. Considering the large decrease of desorption energy with increasing coverage for alkali adsorption systems this result is somewhat surprising. Two different calculations however correctly predict that the frequency remains nearly constant when the coverage is changed [30, 31]. 
On one point our results differ from those of Astaldi et al. [7]. Both for adsorbed $\mathrm{Na}$ and $\mathrm{K}$ they observe a loss peak at twice the loss energy of the peak ascribed by them and by us to adsorbate vibrations. They find the overtone to be relatively strong towards the high coverage end of the coverage range of the observation of loss structure. The authors ascribe the peak to a nonlinear relationship between the induced dipole moment and the distance of the adsorbate above the metal surface. In such case, and as pointed out by the authors, the oscillation could be perfectly symmetric but yet, due to this nonlinearity, there could be strong overtones.

According to our results the frequencies ascribed to overtones are not present when only alkali metal atoms are adsorbed. Our first indication of this was that a peak at around twice the fundamental loss energy appears and grows if the sample is left in the experimental chamber for a few hours (Fig. 4). Looking for possible

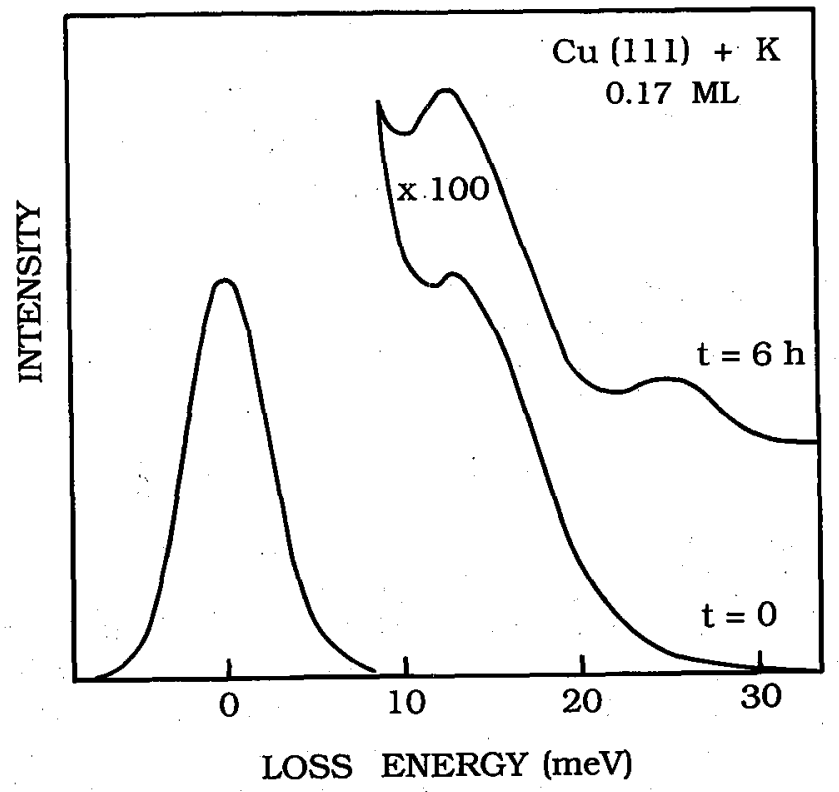

Fig. 4. Electron energy loss spectrum for $\mathrm{K}$ adsorbed on $\mathrm{Cu}(111)$ immediately after the alkali metal deposition and six hours after the deposition.

contaminants we have found that a peak at twice the loss energy of that due to the alkali metal vibrations appears after exposure of the sample to water vapour (Fig. 5): The fact that the peaks observed by Astaldi et al. [7] are due to water vapour exposure of course does not mean that the interesting mechanism suggested by them to account for the observations is wrong. It only means that more work is needed to clarify the origin of the peaks ascribed to overtones. We have noted that the frequency of the fundamental decreases by around $10 \%$ after exposure of the sample to water vapour. This may explain why the frequencies reported by us are a couple of $\mathrm{meV}$ higher than those reported by Astaldi et al. 


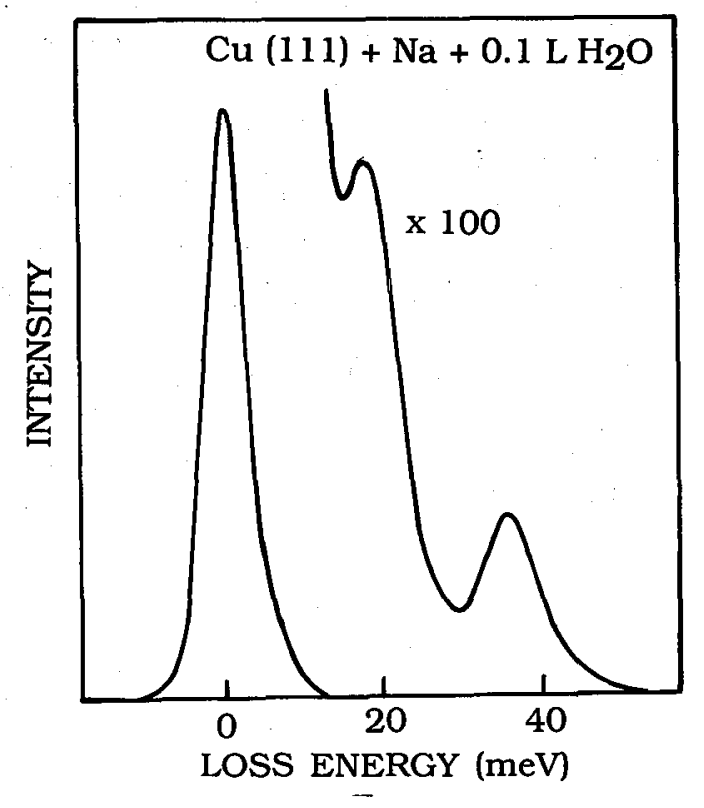

Fig. 5. Electron energy loss spectra for $\mathrm{Na}$ on $\mathrm{Cu}(111)$ at $20 \%$ of full monolayer coverage after exposure of the sample to water vapour. Before the exposure only the peak at around $20 \mathrm{meV}$ loss energy is observed.

\section{3. $S H G$ for Cs monolayers on $\mathrm{Cu}(111)$}

Figure 6 shows the optical second harmonic generation recorded in the specular direction ( $55^{\circ}$ incidence angle) when light from a pulsed ( $\left.8 \mathrm{~ns}, 1 \mathrm{~mJ}\right) \mathrm{Nd}: \mathrm{YAG}$ laser $(\lambda=1064 \mathrm{~nm})$ is incident on a $\mathrm{Cu}(111)$ crystal and the coverage of $\mathrm{Cs}$ is varied. The incident light is p-polarized and the azimuthal angle is chosen such that it is close to the angle giving maximum second harmonic intensity. The very pronounced enhancement produced by the adsorption of monolayer amounts of alkali metal atoms have been observed in previous experiments [32, 33]. In one earlier experiment, for $\mathrm{Rb}$ on $\mathrm{Ag}(110)$, the $\mathrm{SH}$ intensity has furthermore been found to show a strong oscillatory dependence on the overlayer thickness [33]. The oscillations mirror in a very direct way the dynamic Friedel oscillations near inside a metal surface. Similar spectacular oscillations are observed in the present measurements but will not be discussed further here.

Instead we focus attention on the likewise strong intensity variations recorded in the monolayer range of coverages shown in Fig. 6. The absolute thickness scale shown in the diagram is obtained from the evaporation time using as a reference the assumption that maximum photoemission intensity is observed for an overlayer state close below $E_{\mathrm{F}}$ when the coverage is one full monolayer of Cs. At RT the coverage saturates at around $95 \%$ of full monolayer coverage.

The distinct structure of the intensity versus coverage curve in Fig. 6 indi- 


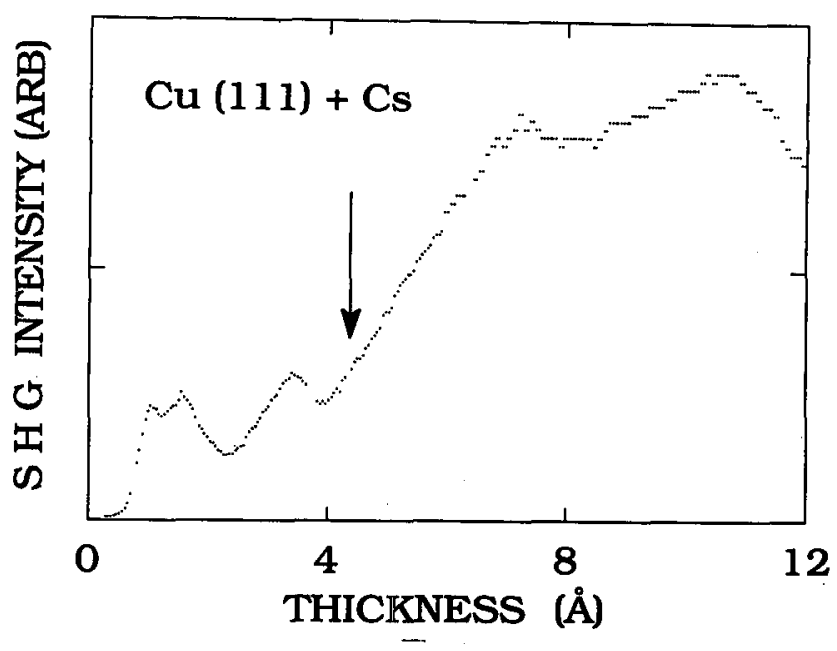

Fig. 6. SHG intensity versus thickness for $\mathrm{Cs}$ on $\mathrm{Cu}(111)$ for $p$-polarized incident light with wavelength $1064 \mathrm{~nm}$ and an incidence angle of $55^{\circ}$. The arrow indicates the thickness corresponding to one close-packed atomic layer of Cs.

cates that the SH intensity is sensitive to particular details of the surface electronic structure and that this changes as the coverage is changed. One advantage of using $\mathrm{Cu}(111) / \mathrm{Cs}$ as sample in exploratory studies of SHG as a surface probe is that the surface electronic structure of this adsorption system is quite well-known. The second advantage is that the surface electronic structure changes considerably when the coverage is changed primarily due to the large change of the work function. Some electronic surface states, the image potential surface states, have energies which shift in energy such that their energy relative to the vacuum level is nearly constant. Ideally to explore SHG as a surface state spectroscopy one would like to have an easily tunable light source. Lacking this an alternative is to use a constant photon energy and a tunable electronic structure like that of $\mathrm{Cs}$ covered $\mathrm{Cu}(111)$.

Figure 7 shows the main features of the surface electronic structure for $k_{\|}=0$ for $\mathrm{Cs}$ covered $\mathrm{Cu}(111)$. For the clean surface there are surface states $0.4 \mathrm{eV}$ below and $4.1 \mathrm{eV}$ above $E_{\mathrm{F}}[34,35]$. When the work function is reduced the state above $E_{\mathrm{F}}$ together with the whole Rydberg-like series of image potential states will shift as indicated. The shift of the $\mathrm{Cu}(111)$ surface state below $E_{\mathrm{F}}$ has been measured earlier [8]. At high monolayer coverage there appears an overlayer state close below $E_{\mathrm{F}}[10]$. The energies of the empty overlayer states may be estimated from a simple phase model for overlayer states [4]. Recently empty overlayer states have been observed via two-photon photoemission [36].

A striking correlation between the surface electronic structure and the measured SHG signal is that at twice the fundamental laser frequency optical transitions become possible at the coverage corresponding to the first SII intensity peak. We therefore associate the observed doublet with the transitions indicated by the 


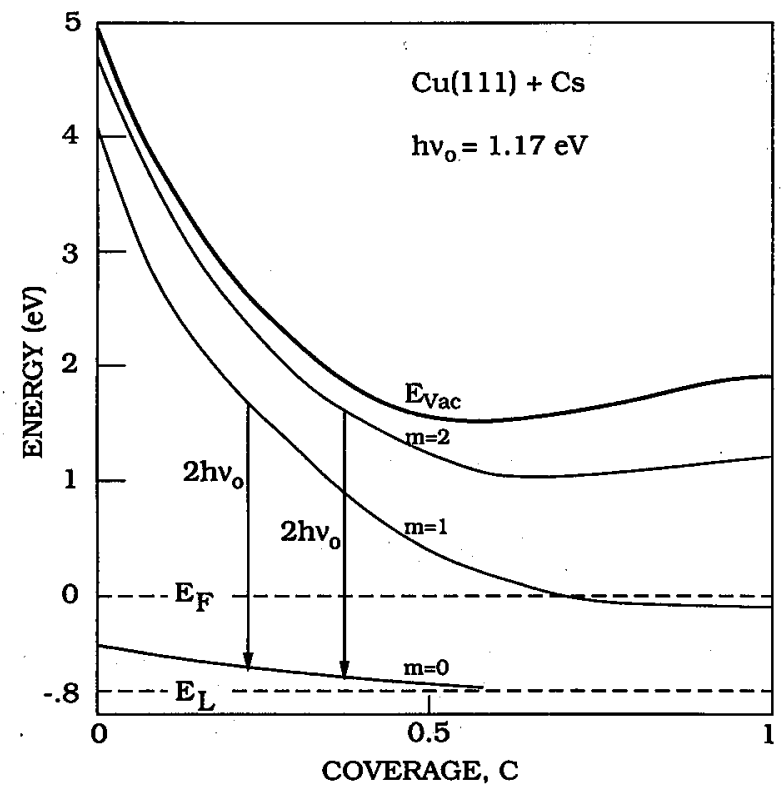

Fig. 7. Energy shifts of surface electronic structure versus Cs coverage for monolayer amounts of the alkali metal on $\mathrm{Cu}(111)$.

arrows in Fig. 7. We associate the third peak (at $3.3 \AA$ in Fig. 6) with the surface barrier absorption peak expected to appear at around $0.8 \omega_{p}$ for free electron like metals [37]. It has previously been found that this surface barrier excitation can be observed for alkali metal monolayers via the photoelectric effect [26] and via the reflectance [38]. It was furthermore shown [39] that the electron gas in the overlayer at high monolayer coverages has a uniform density proportional to the coverage so that $n=n_{\mathrm{Cs}} \Theta$ where $n_{\mathrm{Cs}}$ is the electron density of Cs metal and $\Theta$ is the coverage such that $\Theta=1$ for one close-packed atomic layer. With this one expects a maximum photoabsorption at energy of $0.8 \omega_{p} \sqrt{\Theta}$. Using the free electron value for the plasmon frequency of Cs one obtains a coverage $\Theta=0.85$ in fair agreement with the measured peak (Fig. 6). A full calculation of the photoabsorption of adsorbed Cs monolayers at different coverages have recently been made by Liebsch, Hincelin and Lopes-Rios [38].

The present interpretation may not be unique. The main result is not the details of the interpretation but the observation that the SH intensity can vary strongly with the monolayer coverage and not just in a monotonous manner from one level for the bare substrate to another level for the full monolayer. This shows that the SH is sensitive to the details of the surface electronic structure and we have pointed out details that we believe are relevant for an explanation of the SH data. 


\section{Summary}

We have discussed briefly some recent results obtained with UPS, HREELS and SHG for adsorbed alkali metal monolayers. The main results are that valuable information with UPS for low alkali metal coverages has so far been obtained only for metal substrates with a band gap and with a partly filled surface state in the gap. In these cases on the other hand one has a unique opportunity to monitor the charge exchange between the adsorbate and the substrate via the surface state. For $\mathrm{Na}$ on $\mathrm{Si}(100)$ we have monitored the metallization of the surface region in the monolayer coverage range and found that metal induced gap states can be observed if the sample is kept at low temperature during $\mathrm{Na}$ deposition and measurement. The main conclusion of the HREELS data is that the traditional picture of alkali metal adsorption systems gives the qualitatively correct predictions regarding the observations. The adsorbate vibrates as expected for a particle with a substantial charge which however decreases with increasing coverage and at high monolayer coverage no vibrations are detected due to the metallic character of the adsorbate. SHG is at an earlier stage of development as a surface spectroscopy than the two other techniques discussed in this article. Our results show a lively variation for the SHG signal in the monolayer coverage range and we have pointed out that it is possible to understand this variation in terms of the known surface electronic structure and surface barrier induced photoabsorption.

\section{Acknowledgement}

This work has been supported by the Swedish Natural Science Research Council, the Swedish Board for Technical Development and by the Knut and Alice Wallenberg Foundation.

\section{References}

[1] R.W. Gurney, Phys. Rev. 47, 479 (1935).

[2] N.D. Lang, A.R. Williams, Phys. Rev. B 18, 616 (1978).

[3] S. Holmstrőm, Phys. Scr. 36, 529 (1978).

[4] S.Å. Lindgren, L. Walldén, Surf. Sci. 211/212, 394 (1989).

[5] R.L. Gerlach, T.N. Rhodin, Surf. Sci. 19, 403 (1970).

[6] E.L. Garfunkel, G.A. Somorjai, Surf. Sci. 115, 441 (1982).

[7] C. Astaldi, P. Rudolf, S. Modesti, Solid State Commun. 75, 847 (1990).

[8] S.A. Lindgren, L. Walldén, Solid State Commun. 28, 283 (1978); ibid. 34, 671 (1980).

[9] G.M. Watson, P.A. Bruhwiler, E.W. Plummer, H.J. Sagner, K.H. Frank, Phys. Rev. Lett. 65, 468 (1990).

[10] S.A. Lindgren, L. Walldén, Phys. Rev. Lett. 59, 3003 (1987).

[11] For a review see G.L. Richmond, J.M. Robinson, V.L. Shannon, Prog. Surf. Sci. 28, 1 (1988).

[12] H.B. Nielsen, U. Burghaus, G. Broström, E. Matthias, Surf. Sci. Lett. 234, L271 (1990). 
[13] L. Walldén, T. Gustafsson, Phys. Scr. 6, 73 (1972).

[14] B. Woratschek, W. Sesselmann, J. Kuppers, G. Ertl, H. Haberland, Phys. Rev. Lett. 55, 611 (1985); ibid. 55, 1231 (1985).

[15] S.A. Lindgren, L. Walldén, to be published.

[16] R.C. Jaklevic, J. Lambe, Phys. Rev. B 12, 4146 (1975).

[17] A.L. Wachs, A.P. Shapiro, T.C. Hsieh, T.C. Chiang, Phys. Rev. B 33, 1460 (1986).

[18] T. Miller, A. Samsavar, G.E. Franklin, T.C. Chiang, Phys. Rev. Lett. 61, 1404 (1988).

[19] S.Å. Lindgren, L. Walldén, J. Phys.: Condens. Matter 2, 5929 (1990).

[20] S.A. Lindgren, L. Walldèn, Phys. Rev. Lett. 61, 2894 (1988).

[21] Y. Enta, T. Kinoshita, S. Suzuki, S. Kono, Phys. Rev B 39, 1125 (1989).

[22] Y. Enta, S. Suzuki, S. Kono, T. Sakamoto, Phys. Rev. B 39, 5524 (1989).

[23] M. Tikhov, G. Boishin, L. Surnev, Surf. Sci. 241, 103 (1991).

[24] P. Pervan, E.G. Michel, G.R. Castro, R. Miranda, K. Wandelt, J. Vac. Sci. Technol. A 7, 1885 (1989).

[25] G. Castro, P. Pervan, E.G. Michel, R. Miranda, K. Wandelt, Vacuum 41, 564 (1990).

[26] L., Walldén, Phys. Rev. Lett. 54, 943 (1985).

[27] T. Aruga, H. Tochihara, Y. Murata, Phys. Rev. Lett. 53, 372 (1984).

[28] P. Soukiassian, M.H. Bakshi, Z. Hurych, T.M. Gentle, Surf. Sci. 221, L759 (1989).

[29] S.Å. Lindgren, C. Svensson; L. Walldén, Phys. Rev. B 42, 1467 (1990).

[30] H. Ishida, Phys. Rev. B 39, 5492 (1989).

[31] P.A. Serena, N. Garcia, private communication.

[32] H.W.K. Tom, C.M. Mate, X.D. Zhu, J.E. Crowell, Y.R. Shen, G.A. Somorjai, Surf. Sci. 172, 466 (1986).

[33] K.J. Jong, D. Heskett, H.L. Dai, A. Liebsch, E.W. Plummer, Phys. Rev. Lett. 61, 1380 (1988).

[34] P.O. Gartland, B. Slagsvold, Phys. Rev. B 12, 4047 (1975).

[35] K. Giesen, F. Hage, F.J. Himpsel, H.J. Riess, W. Steinmann, Phys. Rev. B 33, 5241 (1986).

[36] N. Fisher, S. Schuppler, R. Fischer, Th. Fauster, W. Steinmann, Phys. Rev. B 43, 14722 (1991).

[37] P. Feibelman, Prog. Surf. Sci. 12, 287 (1982).

[38] A. Liebsch, G. Hincelin, T. Lopez-Rios, Phys. Rev. B 41, 10463 (1990).

[39] S.Å. Lindgren, L. Walldén, Solid State Commun. 25, 13 (1978); Phys. Rev. B 22, 5967 (1980). 